\title{
Hypofractionated three-dimensional conformal radiotherapy for medically inoperable early stage non-small-cell lung cancer
}

\author{
Joo Ho Lee, MD¹, Hong-Gyun Wu, MD ${ }^{1,2,3}$, Hak Jae Kim, MD¹, Charn II Park, MD', Se-Hoon Lee, MD, \\ Dong-Wan Kim, MD, Dae Seog Heo, MD \\ ${ }^{1}$ Department of Radiation Oncology, ${ }^{2}$ Cancer Research Institute, ${ }^{3}$ Institute of Radiation Medicine, Medical Research Center, \\ ${ }^{4}$ Department of Internal Medicine, Seoul National University College of Medicine, Seoul, Korea
}

Purpose: The purpose of this study was to assess the clinical outcomes of hypofractionated radiotherapy (HFRT) with threedimensional conformal technique for medically inoperable patients with early stage non-small-cell lung cancer (NSCLC) and to evaluate prognostic factors.

Materials and Methods: We performed a retrospective review of 26 patients who underwent HFRT for early stage NSCLC between September 2005 and August 2011. Only clinical stage T1-3NO was included. The median RT dose was 70 Gy (range, 60 to $72 \mathrm{~Gy}$ ) and the median biologically equivalent dose (BED) was $94.5 \mathrm{~Gy}$ (range, 78.0 to $100.8 \mathrm{~Gy}$ ). In 84.6\% of patients, 4 Gy per fraction was used. Neoadjuvant chemotherapy with paclitaxel and cisplatin was given to 2 of 26 patients.

Results: The median follow-up time for surviving patients was 21 months (range, 13 to 49 months). The overall response rate was $53.9 \%$, and the initial local control rate was 100\%. The median survival duration was 27.8 months. Rates of 2-year overall survival, progression-free survival (PFS), local control (LC), and locoregional-free survival (LRFS) were 54.3\%, 61.1\%, 74.6\%, and 61.9\%, respectively. Multivariate analysis showed that $\mathrm{BED}$ (>90 vs. $\leq 90 \mathrm{~Gy}$ ) was an independent prognostic factor influencing PFS, LC, and LRFS. Severe toxicities over grade 3 were not observed.

Conclusion: Radical HFRT can yield satisfactory disease control with acceptable rates of toxicities in medically inoperable patients with early stage NSCLC. HFRT is a viable alternative for clinics and patients ineligible for stereotactic ablative radiotherapy. BED over 90 Gy and 4 Gy per fraction might be appropriate for HFRT.

Keywords: Non-small-cell lung cancer, Hypofractionated radiotherapy, Medically inoperable, Early stage

\section{Introduction}

Surgical resection with lobectomy is the treatment of choice for early stage non-small-cell lung cancer (NSCLC) [1]. However, this surgical option is limited to a small proportion of NSCLC cases due to perioperative mortality, poor medical condition, and patient refusal [1]. Historically, radical radiotherapy has been an alternative option for patients who cannot undergo surgery. However, conventional fractionated radiotherapy with 60 to $66 \mathrm{~Gy}$ in 1.8- or 2-Gy fractions results in poor outcomes with about 50\% local control and about $20 \%$ to $30 \%$ overall survival rate at 3 years $[2,3]$.

Received 6 November 2012, Revised 26 December 2012, Accepted 16 January 2013.

Correspondence: Hong-Gyun Wu, MD, Department of Radiation Oncology, Seoul National University College of Medicine, 101 Daehak-ro, Jongno-gu, Seoul 110-744, Korea. Tel: +82-2-2072-3177, Fax: +82-2-765-3317, E-mail: wuhg@snu.ac.kr

(c) This is an Open Access article distributed under the terms of the Creative Commons Attribution Non-Commercial License (http://creativecommons.org/ licenses/by-nc/3.0/) which permits unrestricted non-commercial use, distribution, and reproduction in any medium, provided the original work is properly cited.

www.e-roj.org 
One way to improve the outcomes of radial radiotherapy alone is to escalate the radiation dose. However, escalating the dose with conventional fractionation leads to protracted treatment, resulting in increased cost, inconvenience, and the risk of tumor repopulation $[4,5]$. In contrast, a hypofractionated regimen can intensify the radiation dose as well as shorten the overall treatment duration. Although the radiobiological benefit of hypofractionation is well known, increases in late toxicities can restrict its use. Three-dimensional conformal radiation therapy (3D-CRT) allows the formulation of a complex multiple-field plan, and makes it possible to protect normal tissue from high doses of radiotherapy, while maintaining target coverage. The recently introduced stereotactic ablative radiotherapy (SABR) regimen yields high local control rates (85-95\%) and acceptable toxicities in early stage NSCLC [6-8].

However, SABR requires levels of precision and accuracy that surpass the requirements of conventionally fractionated radiation therapy or intensity-modulated delivery. Strict protocols must be followed for quality assurance that require expensive treatment equipment and highly qualified human resources [9], which makes it impractical for small-sized cancer centers. Hypofractionated radiotherapy (HFRT) using 3D-CRT can be considered as the second policy in clinics and patients ineligible for SABR. Some studies of HFRT using various radiation dose schedules resulted in improved outcomes beyond those achieved with conventionally fractionated radiotherapy [10-14]. Thus, the purpose of the present study was to assess the clinical outcomes of HFRT for medically inoperable patients with early stage NSCLC and to evaluate prognostic factors.

\section{Materials and Methods}

\section{Patients}

The Institution's Ethical Review Board of the Seoul National University Hospital, Seoul, Korea, approved this retrospective study. Between September 2005 and August 2011, 26 patients underwent HFRT with radical aim for NSCLC at the Seoul National University Hospital, Seoul, Korea. Inclusion criteria were: 1) histologically or clinically diagnosed primary NSCLC; 2) clinical stage T1-3NO (American Joint Committee on Cancer Cancer Staging, 7th edition); 3) completion of hypofractionated radiotherapy with radical aim; and 4 ) inoperable status due to medical contraindication or refusal of surgery. Of the 26
Hypofractionated 3D conformal RT for early stage NSCLC

patients, 23 were considered to be medically inoperable due to medical conditions such as chronic pulmonary disease, advanced age, or other chronic illnesses. The remaining 3 patients refused surgery.

In all patients, the clinical work-up included a detailed medical history, physical examination, complete blood count, liver function test, renal function test, pulmonary function test, and computed tomography (CT) of the chest. All but 2 patients underwent positron emission tomography (PET). The 2 patients who did not undergo PET were examined by bone scan and sonography of abdomen for staging. Patient characteristics are shown in Table 1.

\section{Treatment}

All patients underwent a CT simulation in the treatment position. Tumor motion with breathing was monitored in all patients by fluoroscopy, and the amplitude of tumor motion was considered in RT planning. The delineation and planning for HFRT were CT-based. RT was administered with 6-MV X-ray with 3D-CRT technique using three to six coplanar and noncoplanar beams. The gross tumor volume (GTV) encompassed the radiologically visible tumor on the mediastinal setting of simulation CTs. The contoured GTV was also modified with consideration of PET and CT lung window. Clinical target volume comprised GTV plus 10-15 mm anisotropic margins, and additional margins from clinical target volume were not adopted for planning target volume. Elective nodal irradiation was not adopted in all patients.

The radiation dose ranged from 60 to $72 \mathrm{~Gy}$ (median, $70 \mathrm{~Gy}$ ) and daily fraction sizes were $4 \mathrm{~Gy}, 3.13 \mathrm{~Gy}$, and $3 \mathrm{~Gy}$ in $84.6 \%$, $7.7 \%$, and $7.7 \%$ of patients, respectively. To compare the effects of various protocols with different fraction sizes and total doses, a biologically equivalent dose (BED) was adopted in a linear quadratic model. The $\alpha / \beta$ ratio was assumed to be 10 for acute effects on normal tissues and tumors. BED ranged from 78.0 to 100.8 Gy (median, 94.5 Gy). BED > 90 Gy was reported in $13 / 26$ patients.

The median PTV was $125.3 \mathrm{~mL}$ (range, 11.5 to $450.5 \mathrm{~mL}$ ) and the median GTV was $22.5 \mathrm{~mL}$ (range, 3.9 to $166.8 \mathrm{~mL}$ ). The median V20 of both lungs was 15.9\% (range, 6.5\% to 28.2\%). The maximal dose to the spinal cord ranged from 4 to $37 \mathrm{~Gy}$. The V50 of the esophagus was below 5\% in all but one patient. Neoadjuvant chemotherapy with paclitaxel and cisplatin was administered to 2/26 patients. The other 24 patients received radical radiotherapy alone. 


\section{Evaluation of initial clinical response and toxicity}

In terms of response criteria, a complete response (CR) was defined as disappearance of all measurable disease and the absence of new lesions for at least 4 weeks. For measurable disease, a partial response (PR) was defined as a 4-week reduction of greater than $30 \%$ of the sum of the diameters of the cross-sectional diameters of all measurable lesions. Progressive disease (PD) was defined as either a 4-week increase of greater than $20 \%$ of the sum of the diameters of the cross-sectional diameters of all assessable lesions or the appearance of new lesions. Stable disease (SD) was defined

Table 1. Patient and treatment characteristics

\begin{tabular}{|c|c|}
\hline Characteristic & Value $(\%)$ \\
\hline Age (yr), median (range) & $76(65-83)$ \\
\hline \multicolumn{2}{|l|}{ Gender } \\
\hline Male & $23(88.5)$ \\
\hline Female & $3(11.5)$ \\
\hline \multicolumn{2}{|l|}{ ECOG performance } \\
\hline 0 & $1(3.8)$ \\
\hline 1 & $16(61.5)$ \\
\hline 2 & $9(34.7)$ \\
\hline FEV1, mean (range) & $1.6(0.8-2.5)$ \\
\hline FEV1\% predicted, mean (range) & $77.3(35.0-157.0)$ \\
\hline \multicolumn{2}{|l|}{ T stage } \\
\hline $\mathrm{T} 1$ & 5 (19.2) \\
\hline $\mathrm{T} 2$ & $16(61.5)$ \\
\hline T3 & 5 (19.3) \\
\hline \multicolumn{2}{|l|}{ Pathology } \\
\hline Adenocarcinoma & 5 (19.2) \\
\hline Squamous cell carcinoma & $12(46.2)$ \\
\hline Others & $6(23.1)$ \\
\hline Not verified & $3(11.5)$ \\
\hline \multicolumn{2}{|l|}{ Location of tumor } \\
\hline Central & $8(30.8)$ \\
\hline Peripheral & 18 (69.2) \\
\hline GTV (mL), mean (range) & 45.9 (3.9-166.8) \\
\hline PTV (mL), mean (range) & $165.9(11.5-450.5)$ \\
\hline RT dose (Gy), median (range) & $70(60-72)$ \\
\hline BED (Gy), median (range) & $94.5(78.0-100.8)$ \\
\hline \multicolumn{2}{|l|}{ Fraction size (Gy) } \\
\hline 3 & $2(7.7)$ \\
\hline 3.13 & $2(7.7)$ \\
\hline 4 & $22(84.6)$ \\
\hline Neoadjuvant chemotherapy & $2(7.7)$ \\
\hline
\end{tabular}

ECOG, the Eastern Cooperative Oncology Group; FEV1, forced expiratory volume in 1 second; GTV, gross tumor volume; PTV, planning target volume; RT, radiotherapy; BED, biologically equivalent dose using a linear quadratic model, and the $\alpha / \beta$ ratio was 10 for acute effects on normal tissues and tumors. when there was neither sufficient shrinkage to qualify for PR nor sufficient increase to qualify for PD [15]. Acute and late toxicities were evaluated according to the Radiation Therapy Oncology Group/European Organization for the Treatment of Cancer scale [16].

\section{Statistical analysis}

Overall survival (OS), progression-free survival (PFS), local control (LC), and locoregional-free survival (LRFS) were calculated as the interval from the first date of treatment to the date of death or last follow-up, any progression detection, tumor progression within the radiation portal, and any failure within the regional lymph node stations and ipsilateral lung.

Survival curves were generated by the Kaplan-Meier method and univariate survival comparisons were performed using the log-rank test. Multivariate analyses were conducted using a Cox proportional hazards model with a backward stepwise selection procedure. A p-value of $<0.05$ indicated statistical significance.

\section{Results}

The median follow-up time for surviving patients was 21 months (range, 13 to 49 months). The mean overall treatment duration was 24 days. CR occurred in 2 patients (7.7\%), PR in $12(46.2 \%)$, SD in $9(34.6 \%)$. PD due to distant metastasis was observed in 3 patients (11.5\%). The overall response rate was $53.9 \%$, and the initial local control rate was 100\%. The median OS of all patients was 27.8 months. Two-year OS, PFS, LC and LRFS were $54.3 \%, 61.1 \%, 74.6 \%$, and $61.9 \%$, respectively. Relapses after treatment occurred in 9 patients during the follow-up period. The initial sites of failure were as follows: 3 local only, 2 regional only, 1 local and regional, and 3 local and distant.

Univariate analysis of prognostic factors on clinical outcome is shown in Table 2. Age (>75 vs. $\leq 75$ years) was a significant prognostic factor correlated with OS. BED (>90 vs. $\leq 90 \mathrm{~Gy}$ ) was significantly related to PFS, LC, and LRFS. Fig. 1 illustrates the PFS and LC curves for each group. T stage was also a significant prognostic factor in LC. In multivariate analysis, BED (>90 vs. $\leq 90$ Gy) was an independent prognostic factor predicting PFS $(p=0.025$; hazard ratio $[H R], 6.04 ; 95 \%$ confidence interval $[\mathrm{Cl}], 1.25$ to 29.2), LC ( $p=0.046$; HR, 9.27; $95 \% \mathrm{Cl}, 1.04$ to 82.9 ), and LRFS ( $p=0.022 ; \mathrm{HR}, 6.29 ; 95 \%$ $\mathrm{Cl}, 1.30$ to 30.38 ). No factors significant to $0 \mathrm{~S}$ were found in multivariate analysis. 
Table 2. Univariate analysis of factors affecting clinical outcome

\begin{tabular}{|c|c|c|c|c|c|c|c|c|}
\hline & $2-y r O S$ & $p$-value & $2-y r$ PFS & $p$-value & 2-yr LC & $p$-value & $2-y r$ LRFS & $p$-value \\
\hline \multicolumn{9}{|l|}{ Age (yr) } \\
\hline$\leq 75$ & 81.8 & 0.031 & 76.2 & 0.111 & 88.9 & 0.233 & 76.2 & 0.128 \\
\hline$>75$ & 35.0 & & 51.9 & & 62.5 & & 54.2 & \\
\hline \multicolumn{9}{|l|}{ ECOG } \\
\hline 0 or 1 & 64.3 & 0.137 & 75.0 & 0.609 & 84.4 & 0.348 & 68.1 & 0.358 \\
\hline 2 & 45.7 & & 42.9 & & 53.6 & & 47.6 & \\
\hline \multicolumn{9}{|l|}{ FEV1 } \\
\hline$\leq 1.5$ & 0.583 & 0.948 & 78.8 & 0.587 & 87.5 & 0.255 & 80.8 & 0.531 \\
\hline$>1.5$ & 0.536 & & 50.5 & & 65.7 & & 50.5 & \\
\hline \multicolumn{9}{|l|}{ T stage } \\
\hline $\mathrm{T} 1$ & 80.0 & 0.688 & 80.0 & 0.277 & 80.0 & 0.045 & 80.0 & 0.258 \\
\hline T2 & 49.2 & & 76.4 & & 90.9 & & 78.1 & \\
\hline T3 & 60.0 & & 20.0 & & 40.0 & & 20.0 & \\
\hline \multicolumn{9}{|l|}{ Pathology } \\
\hline Adenocarcinoma & 60.0 & 0.912 & 100 & 0.330 & 100 & 0.463 & 100 & 0.290 \\
\hline Squamous cell carcinoma & 57.1 & & 62.9 & & 62.9 & & 62.9 & \\
\hline Others & 62.5 & & 41.7 & & 50.0 & & 41.7 & \\
\hline \multicolumn{9}{|l|}{ Location of tumor } \\
\hline Central & 31.3 & 0.687 & 52.5 & 0.714 & 52.5 & 0.159 & 52.5 & 0.667 \\
\hline Peripheral & 66.2 & & 64.9 & & 84.4 & & 65.7 & \\
\hline \multicolumn{9}{|l|}{$\mathrm{GTV}(\mathrm{mL})$} \\
\hline$\leq 20$ & 64.3 & 0.137 & 75.0 & 0.609 & 82.5 & 0.350 & 75.0 & 0.660 \\
\hline$>20$ & 45.7 & & 42.9 & & 64.3 & & 43.8 & \\
\hline \multicolumn{9}{|l|}{ Radiation dose (BED, Gy) } \\
\hline$\leq 90$ & 53.0 & 0.448 & 34.1 & 0.011 & 56.8 & 0.036 & 34.1 & 0.009 \\
\hline$>90$ & 55.0 & & 81.5 & & 88.9 & & 82.1 & \\
\hline
\end{tabular}

Values are presented as percentage of patient. A p-value was analyzed by log rank test.

OS, overall survival; PFS, progression-free survival; LC, local control; LRFS, locoregional-free survival; ECOG, the Eastern Cooperative Oncology Group; FEV1, forced expiratory volume in 1 second; GTV, gross tumor volume; BED, biologically equivalent dose using a linear quadratic model, and the $\alpha / \beta$ ratio was 10 for acute effects on normal tissues and tumors.

A

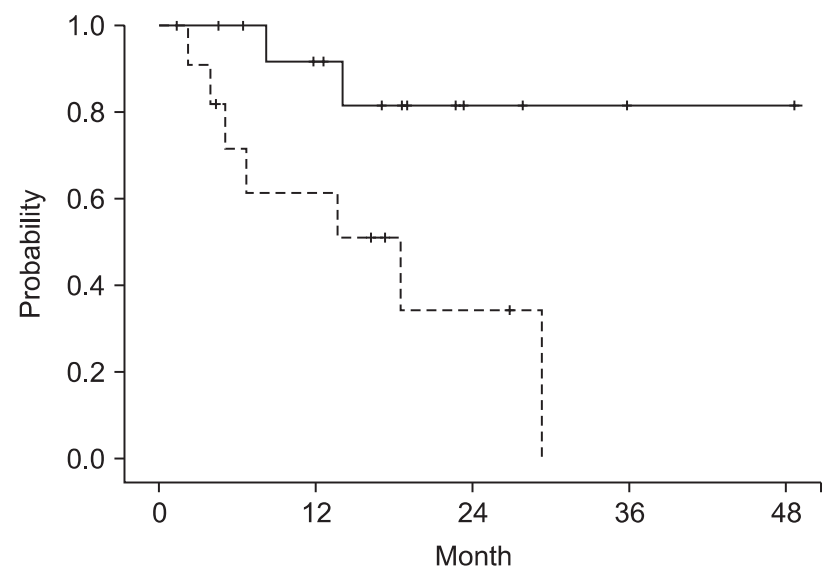

B

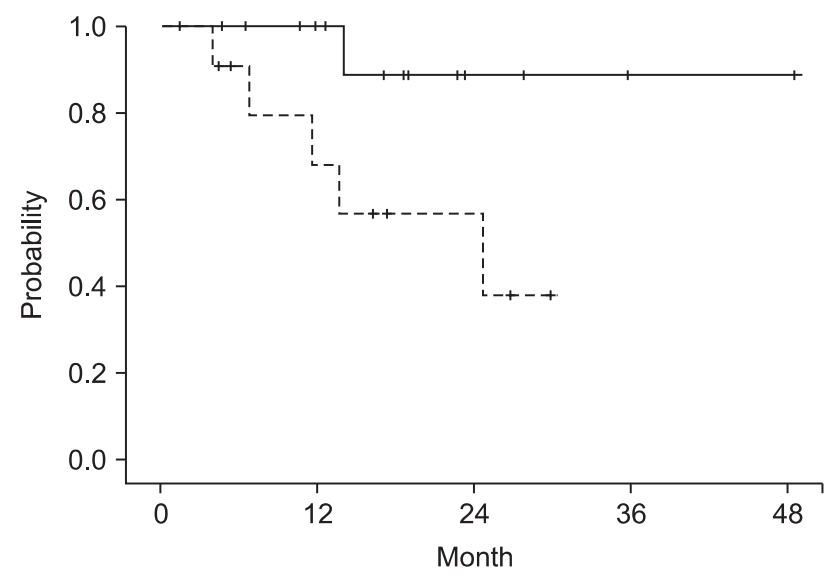

Fig. 1. (A) Progression-free survival and (B) local control between the subgroups with biologically equivalent dose (BED) using a linear quadratic model, and the $\alpha / \beta$ ratio was 10 for acute effects on normal tissues and tumors. Solid line, BED > 90 Gy; dotted line, BED $\leq$ $90 \mathrm{~Gy}$. 
Among acute toxicities, grade 2 lung toxicities occurred in one patient and grade 2 esophagus toxicity occurred in one patient. Among late toxicities, one patient experienced grade 2 late lung toxicity, and one patient experienced grade 2 esophagus toxicity (stricture). V50 and maximal dose of esophagus in the patient reporting grade 2 esophageal toxicity was 38.6\% and $62 \mathrm{~Gy}$, respectively. The fraction size and BED in the patient reporting grade 2 esophageal toxicity were $4 \mathrm{~Gy}$ and $100 \mathrm{~Gy}$, respectively. No patient showed grade 3 toxicity in the acute or late phase.

\section{Discussion and Conclusion}

The purpose of this study was to evaluate the clinical outcomes of HFRT for medically inoperable patients with early stage NSCLC and to evaluate prognostic factors. A 2-year OS of 54.3\%, 2-year PFS of 61.1\%, 2-year LC of 74.6\%, and 2-year LRFS of $61.9 \%$ were achieved; this outcome was comparable with results from previous HFRT reports (2-year OS of 50$60 \%$, 2-year LC of $63-76 \%$ ), but PFS was superior to those reported in previous studies (2-year PFS of 38-40\%) [1014]. Local failure was the predominant pattern, as in previous studies of radical radiotherapy in early stage $\operatorname{NSCLC}[3,11,17]$. The patterns of failure in these patient groups emphasize the importance of local control. For the control of NSCLC, it has been suggested that an overall treatment period shorter than kick-off time (Tk) (less than 4 weeks) has advantages over simple dose escalation with conventional fractions [10]. SABR with a large fraction size can achieve favorable local control for early stage NSCLC, which makes it the current standard treatment for medically inoperable patients $[1,6-8]$.

However, SABR requires highly intensive and qualified resources in terms of all human, physical, and financial aspects [9]. In a 2011 survey in the United States, 46.1\% of responding physicians did not use SABR and only 39\% of small hospitals with one physician adopted SABR [18]. A survey in Japan also reported that $44 \%$ of replying institutions did not utilize SABR [19]. The ability to transfer patients to other hospitals far from patient residences is limited in cases of poor medical status. Although the number of hospitals using SABR is increasing, alternatives to $S A B R$ are necessary for practical reasons. In addition, for patients with large or centrally located tumors SABR may be limited by risk of toxicities [20]. In the current study, 8 patients with central tumors received HFRT. Among them, acute and late grade 2 esophageal toxicity were reported in 1 patient who received high dose HFRT with 4 Gy per fraction and BED $100 \mathrm{~Gy}$. Although total dose and fraction size of HFRT should be adjusted for centrally located or large tumor, HFRT can be the second policy in patients ineligible for SABR.

Although HFRT is a reasonable option, radiotherapy fractions and total doses are very heterogeneous among the studies. Most fraction sizes are 3-3.5 Gy/fraction $[10,11,13]$ and the results are comparable to those of the present study. However Sandhu et al. [12] used 2.2-2.69 Gy/fractions in most patients $(84.8 \%)$ and the resulting local control with a median value of 21.2 months was inferior to those reported in other studies [10-14]. In the current study, 4 Gy/fraction was utilized in $84.6 \%$ of patients, and the toxicity profile was comparable to those reported in previous studies using 3-3.5 Gy/fractions. Cheung et al. [14] also showed that 4 Gy/fraction can be delivered safely with comparable tumor control rates. More accelerated fractionation with $4 \mathrm{~Gy} /$ fraction would lead to additional benefits in terms of tumor repopulation. In addition, radiotherapy is performed on an outpatient basis by most clinics, and the convenience and cost savings resulting from more accelerated radiation schedules with 4 Gy/fractions have practical advantages for patients susceptible to poor performance and comorbidities.

Total dose was also a key factor to define in HFRT. The current study demonstrated that BED over $90 \mathrm{~Gy}$ is an independent significant prognostic factor in LC and PFS using a linear quadratic model, and an $\alpha / \beta$ ratio of 10 to determine acute effects on normal tissues and tumors. In previous studies using conventional fractionation, improved local PFS was observed for a subgroup of patients with no nodal disease receiving $>73$ Gy 3D-CRT [21]. Onishi et al. [8] also demonstrated that BED $\geq 100 \mathrm{~Gy}$ in SABR resulted in better LC and survival outcomes. These results are similar to the dose-response relationship shown in the present study. In terms of safety, no severe toxicities over grade 3 were observed in the present study, and low incidence of toxicities was also reported by Thirion et al. [13] (BED of HFRT, 93.6 Gy). Accordingly, HFRT with high BED over 90 Gy may improve tumor control without increasing toxicities, similar to SABR.

Interestingly, the PFS of the present study was superior to that of previous studies of HFRT [10-14]. The current study used PET for staging in $92.3 \%$ of patients, making this study distinct from previous studies [10-14]. It may be valuable to perform PET for pretreatment evaluation in NSCLC with clinically negative lymph nodes, because occult distant metastasis can be detected by whole body PET, and the 
negative predictive value for mediastinal lymph node is as high as 95\% [22]. The benefits of LC after HFRT may improve PFS due to pretreatment work-up. However, interpretations of our results are limited because by the nature of small retrospective analysis. Our sample was small in part due to the limitations of HFRT. Therefore, the specific radiation dose schedule recommended in this paper should be interpreted with caution. However, HFRT is currently considered an alternative to SABR, so large prospective trials of HFRT might be difficult to perform.

HFRT with radical aim can yield satisfactory disease control with acceptable rates of toxicity in medically inoperable patients with early stage NSCLC. Although the resulting tumor control was inferior to that achieved by SABR, HFRT can be an alternative option for clinics and patients ineligible for SABR. BED over 90 and 4 Gy per fraction may be appropriate for HFRT, but the optimal radiation dose of HFRT should be confirmed by additional large-scale studies.

\section{Conflict of Interest}

No potential conflict of interest relevant to this article was reported.

\section{References}

1. Scott WJ, Howington J, Feigenberg S, Movsas B, Pisters K; American College of Chest Physicians. Treatment of non-small cell lung cancer stage I and stage II: ACCP evidence-based clinical practice guidelines (2nd edition). Chest 2007;132(3 Suppl):234S-42S.

2. Kaskowitz L, Graham MV, Emami B, Halverson KJ, Rush C. Radiation therapy alone for stage I non-small cell lung cancer. Int J Radiat Oncol Biol Phys 1993;27:517-23.

3. Qiao X, Tullgren 0 , Lax I, Sirzen F, Lewensohn R. The role of radiotherapy in treatment of stage I non-small cell lung cancer. Lung Cancer 2003:41:1-11.

4. Fowler JF, Chappell R. Non-small cell lung tumors repopulate rapidly during radiation therapy. Int J Radiat Oncol Biol Phys 2000;46:516-7.

5. Bradley J, Graham MV, Winter K, et al. Toxicity and outcome results of RTOG 9311: a phase I-II dose-escalation study using three-dimensional conformal radiotherapy in patients with inoperable non-small-cell lung carcinoma. Int J Radiat Oncol Biol Phys 2005;61:318-28.

6. Kim W, Kim HJ, Park JH, Huh HD, Choi SH. Treatment results of CyberKnife radiosurgery for patients with primary or recurrent
Hypofractionated 3D conformal RT for early stage NSCLC

non-small cell lung cancer. J Korean Soc Ther Radiol Oncol 2011;29:28-35.

7. Timmerman R, Paulus $R$, Galvin J, et al. Stereotactic body radiation therapy for inoperable early stage lung cancer. JAMA 2010;303:1070-6.

8. Onishi H, Araki T, Shirato H, et al. Stereotactic hypofractionated high-dose irradiation for stage I nonsmall cell lung carcinoma: clinical outcomes in 245 subjects in a Japanese multiinstitutional study. Cancer 2004;101:1623-31.

9. Potters L, Kavanagh B, Galvin JM, et al. American Society for Therapeutic Radiology and Oncology (ASTRO) and American College of Radiology (ACR) practice guideline for the performance of stereotactic body radiation therapy. Int J Radiat Oncol Biol Phys 2010;76:326-32.

10. Bonfili $P$, Di Staso M, Gravina GL, et al. Hypofractionated radical radiotherapy in elderly patients with medically inoperable stage I-II non-small-cell lung cancer. Lung Cancer 2010;67:81-5.

11. Faria SL, Souhami L, Portelance $L$, et al. Absence of toxicity with hypofractionated 3-dimensional radiation therapy for inoperable, early stage non-small cell lung cancer. Radiat Oncol 2006;1:42.

12. Sandhu AP, Messer K, Fuster MM, et al. Hypofractionated radiotherapy for stage I non-small cell lung carcinoma in patients aged 75 years and older. J Cancer Ther 2011;2:167-71.

13. Thirion $P$, Holmberg $O$, Collins $C D$, et al. Escalated dose for non-small-cell lung cancer with accelerated hypofractionated three-dimensional conformal radiation therapy. Radiother Oncol 2004;71:163-6.

14. Cheung PC, Yeung LT, Basrur V, Ung YC, Balogh J, Danjoux CE. Accelerated hypofractionation for early-stage non-small-cel lung cancer. Int J Radiat Oncol Biol Phys 2002;54:1014-23.

15. Eisenhauer EA, Therasse P, Bogaerts J, et al. New response evaluation criteria in solid tumours: revised RECIST guideline (version 1.1). Eur J Cancer 2009;45:228-47.

16. Cox JD, Stetz J, Pajak TF. Toxicity criteria of the Radiation Therapy Oncology Group (RTOG) and the European Organization for Research and Treatment of Cancer (EORTC). Int J Radiat Oncol Biol Phys 1995;31:1341-6.

17. Jeremic B, Classen J, Bamberg M. Radiotherapy alone in technically operable, medically inoperable, early-stage (I/II) non-small-cell lung cancer. Int J Radiat Oncol Biol Phys 2002; 54:119-30.

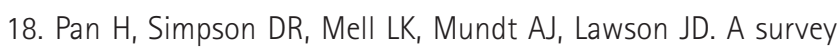
of stereotactic body radiotherapy use in the United States. Cancer 2011;117:4566-72.

19. Nagata Y, Hiraoka M, Mizowaki T, et al. Survey of stereotactic body radiation therapy in Japan by the Japan 3-D Conformal External Beam Radiotherapy Group. Int J Radiat Oncol Biol 
Phys 2009;75:343-7.

20. Song SY, Choi W, Shin SS, et al. Fractionated stereotactic body radiation therapy for medically inoperable stage I lung cancer adjacent to central large bronchus. Lung Cancer 2009;66:8993.

21. Martel MK, Strawderman M, Hazuka MB, Turrisi AT, Fraass BA,
Lichter AS. Volume and dose parameters for survival of nonsmall cell lung cancer patients. Radiother Oncol 1997;44:23-9. 22. Pieterman RM, van Putten JW, Meuzelaar JJ, et al. Preoperative staging of non-small-cell lung cancer with positron-emission tomography. N Engl J Med 2000;343:254-61. 